\title{
Uso de Internet y prácticas políticas de los jóvenes españoles
}

\author{
Spanish young people's Internet use and political practices \\ Eva Espinar-Ruiz / eva.espinar@ua.es \\ Universidad de Alicante, España \\ María José González-Río / mj.gonzalez@ua.es \\ Universidad de Alicante, España
}

\begin{abstract}
In this article we apply a secondary analysis on the micro-data provided by the study 2,889 of the Spanish Center for Sociological Research. The main objective is to observe if there is a correlation between young people's Internet use and their political practices. Specifically, the analyzed variables allow us to study three important aspects: 1) the correlation between the degree of political participation and the frequency of Internet use, 2) the relation between online and offline political participation, and 3) the existence of differences in online political participation with respect of socio-demographic variables. Among the results we can highlight the existence of a statistically significant relation between a higher frequency of Internet use and a greater online and offline political involvement.
\end{abstract}

Key words: young people, Internet, political participation, Spain, quantitative analysis.

Resumen: A partir de un análisis secundario de los microdatos procedentes del estudio 2.889 del Centro de Investigaciones Sociológicas de España, pretendemos observar la posible relación entre el uso de Internet por parte de los jóvenes y sus prácticas políticas. En concreto, las variables disponibles permiten estudiar tres aspectos fundamentales: si existe relación entre la frecuencia de uso de Internet y la participación política; si el grado de participación política en el espacio virtual se correlaciona con la participación fuera de este espacio; y si se observan diferencias en términos de participación política online según variables sociodemográficas. Entre los resultados obtenidos podemos destacar la existencia de una relación estadísticamente significativa entre una mayor frecuencia de uso de Internet y una mayor implicación política tanto en el espacio virtual como offline.

Palabras clave: jóvenes, Internet, participación política, España, análisis cuantitativo. 


\section{Introducción}

El desinterés, la apatía y el desencanto parecen haberse convertido en rasgos que caracterizan la relación con la política de gran parte de los ciudadanos a nivel mundial (Bauman, 2007). Aunque esta actitud estaría afectando a todas las edades, son los jóvenes quienes aparentemente se muestran más alejados de la política, al menos en su dimensión institucional (Mindich, 2005; Banaji y Buckingham, 2010). En esta línea encontramos autores que hablan de una creciente actitud individualista y cínica de los jóvenes hacia la política y llegan a señalarlos como los abanderados de una creciente crisis de la democracia (Furlong y Cartmel, 2007).

Sin embargo, otros estudios cuestionan esta supuesta desafección y falta de participación política de los jóvenes, indicando que los autores que sostienen tales afirmaciones se centran en exceso en las opciones tradicionales e institucionales de hacer política (Bakker y de Vreese, 2011; Coffé y Bolzendahl, 2010). Su tesis es que las interpretaciones que hacen los jóvenes de la política y de las formas de participar en ella difieren de las sostenidas tradicionalmente (Bennett, 2012; Loader et al., 2014). En este nuevo escenario suele destacarse el papel crucial de las tecnologías de la información y la comunicación y, en general, de todas las herramientas que ofrece Internet (Livingstone, 2007; Castells, 2012).

El uso político de la tecnología digital se ha puesto especialmente de relieve a raíz de las movilizaciones ciudadanas que han tenido lugar en los últimos años. Desde las primeras revueltas tunecina y egipcia en contra de regímenes autocráticos, se ha ido produciendo una extensión creciente de la ocupación del espacio público en muy diferentes partes del mundo. En el contexto español, el movimiento de los "indignados" ha puesto sobre la mesa la brecha existente entre la legitimidad política y la legitimidad social. Los jóvenes han estado en la vanguardia de estas movilizaciones haciendo un amplio uso de las nuevas tecnologías (Sloam, 2014).

El correo electrónico, las redes sociales, los blogs y las páginas web han incrementado los recursos de los activistas demostrando una gran eficacia como difusores de iniciativas y como herramientas de movilización (Castells, 2012). Este contexto ha colaborado en promover un creciente interés por el análisis de la participación política de los jóvenes y el impacto de las nuevas tecnologías; si bien, como se verá más adelante, los resultados obtenidos hasta la fecha no permiten hablar de acuerdos unánimes (Hirzalla et al., 2011).

En este artículo, nuestro propósito principal es analizar el uso político que hacen los jóvenes de Internet. Para ello, se ha llevado a cabo un análisis 
secundario de los microdatos procedentes del estudio 2.889 del Centro de Investigaciones Sociológicas (CIS), organismo autónomo dependiente del Ministerio de la Presidencia del Gobierno de España. Esta encuesta se aplicó, en mayo de 2011, a una muestra de jóvenes, de ambos sexos, residentes en España, con edades comprendidas entre los 15 y los 29 años y, aunque orientada al estudio genérico de las nuevas tecnologías, incluye una serie de variables referidas a su utilización política. En concreto, permite analizar tres aspectos relevantes: si existe relación entre la frecuencia del uso de Internet y la participación política; si el grado de participación política en el espacio virtual se correlaciona con la participación fuera de ese espacio; y si existen diferencias significativas en términos de participación política online según variables sociodemográficas.

\section{Internet y la participación política de los jóvenes}

Los estudios empíricos existentes muestran importantes desacuerdos y resultados contradictorios respecto al impacto político de Internet (Borge et al., 2012; De Marco y Robles, 2012). Estas contradicciones se observan tanto en los primeros estudios como en los publicados recientemente, que incorporan el análisis de las redes sociales y la web 2.0 (Xenos et al., 2014). Así, desde las posiciones más optimistas, diversos autores sugieren la existencia de una relación positiva entre el uso de Internet y diferentes indicadores de implicación política (Bode, 2012; Valenzuela et al., 2009; Zhang et al., 2013). Estos estudios destacan la capacidad movilizadora de Internet y su potencialidad para reducir las desigualdades existentes en materia de participación política, llegando a implicar a individuos previamente inactivos o que no comparten el perfil del participante tradicional (Morris y Morris, 2013).

Frente a estos resultados, desde las posturas más pesimistas, algunos autores llegan a plantear la inexistencia de relación o, en todo caso, una relación muy débil entre el uso de Internet y una mayor implicación política (Baumgargner y Morris, 2009; Dimitrova y Bystrom, 2013). Sin embargo, la literatura más abundante se sitúa en una postura que podemos definir de optimismo cauto. Así, encontramos estudios que hablan de relaciones positivas entre el uso de Internet y la participación política, pero limitando tal relación a usos específicos de la Red, a formas concretas de participación política, o a la necesaria interacción de diferentes variables y factores (Gil de Zúñiga et al., 2012; Vitak et al., 2011; Xenos et al., 2014).

En este apartado podemos situar a aquellos autores que defienden la hipótesis de la normalización o el refuerzo, según la cual Internet tiene un 
efecto especialmente positivo entre los ciudadanos políticamente ya comprometidos, mientras que su efecto es mínimo entre los indiferentes (Scheufele y Nisbet, 2002; Banaji y Buckingham, 2010). Estas investigaciones vienen a señalar que los efectos políticos de Internet pueden suponer la reproducción o incluso el incremento de las desigualdades previamente existentes en términos de implicación y participación política (Schlozman et al., 2010).

En este mismo marco de desacuerdos se ubican los estudios que analizan, específicamente, el uso de Internet por parte de la población joven y sus efectos en términos de participación política. Así, entre los resultados obtenidos, es posible encontrar argumentos que respaldan tanto la hipótesis normalizadora (Macafee y De Simone, 2012; Baumgartner y Morris, 2010; Banaji y Buckingham, 2010) como la movilizadora (Zhou y Pinkleton, 2012; Livingstone, 2010; Sloam, 2012), o una combinación de ambas posturas (Nam, 2012; Vissers y Stolle, 2014). Parte de esta aparente ausencia de acuerdos puede explicarse por las diferencias metodológicas y las discrepancias teóricas entre las investigaciones (Xenos et al., 2014).

Por ejemplo, la mayoría de los estudios se han basado en encuestas sobre muestras específicas (como estudiantes universitarios o una limitada franja de edad) o se han centrado en un acontecimiento concreto. Por otra parte, se han aplicado diversas estrategias para definir tanto el uso de Internet como el concepto de participación política. Entonces, mientras que algunos estudios analizan de forma genérica la utilización de Internet o las redes sociales virtuales (Bode, 2012; Zhang et al., 2013), otros inciden en la importancia de distinguir entre los diferentes usos y herramientas que ofrece la red, dado que cada uno de ellos supone distintas implicaciones en términos políticos (Gil de Zúñiga et al., 2012; Yamamoto y Kushin, 2014). En esta línea, algunos autores coinciden en destacar los efectos positivos derivados del uso de Internet como fuente de información (Ekström et al., 2014; Bakker y de Vreese, 2011; Lee et al., 2013), frente a otras opciones como las propias redes sociales (Ekström et al., 2014; Vissers y Stolle, 2014).

Respecto al concepto de participación política, mientras que algunos trabajos se centran en formas de participación tradicionales e institucionales, otros amplían el enfoque para incluir una mayor gama de actividades y optan por conceptos más generales como el de implicación política, compromiso cívico u orientación pública (Anduiza et al., 2010; Dahlgren, 2011; Xenos et al., 2014; Ekström et al., 2014). En este sentido, es posible encontrar diversas formas de diferenciar e interpretar las actividades políticas virtuales frente a las opciones, tradicionales o novedosas, de implicación política offline (Xenos et al., 2014). Por ejemplo, frente a algunos autores que adoptan una 
postura abiertamente crítica con las actividades virtuales, consideradas excesivamente simples y sin efectos relevantes (Morozov, 2009), otros apuestan por destacar la importancia de tales estrategias (Anduiza et al., 2010; Gil de Zúñiga et al., 2010).

Este debate terminológico resulta especialmente significativo en el caso de la población joven. Junto a los estudios que destacan su desafección con las formas clásicas e institucionales de participación política, algunas investigaciones señalan que los jóvenes están desarrollando formas diferentes de relacionarse con la política y la vida pública (Bennett, 2012; Bennett et al., 2011). Desde estos planteamientos se rechaza la calificación de los jóvenes como políticamente apáticos (Berlinguer y Martínez, 2014; Loader et al., 2014; Sloam, 2014), si bien su interés no se manifiesta a través de las opciones tradicionales de participación (voto, militancia en partidos y sindicatos, lectura de prensa, etc.), sino, en gran medida, a través de formas relativamente novedosas como el voluntariado, demostraciones públicas, el boicot al consumo de determinados productos, la participación en movimientos sociales o el activismo virtual.

En este sentido, y en términos generales, buena parte de las investigaciones permiten hablar de un optimismo cauto (Dahlgren, 2011) en relación con las potencialidades políticas de Internet para los jóvenes usuarios. Así, aunque las variables socioeconómicas siguen mostrándose relevantes a la hora de explicar las diferencias en términos de compromiso político (Schlozman et al., 2010; Xenos et al., 2014; Vissers y Stolle, 2014), estudios como el de Scholzman et al. (2010) concluyen que el uso de Internet sí parece reducir las brechas tradicionales en razón de edad. Igualmente, investigaciones como la de Xenos et al. (2014) sugieren la existencia de una relación positiva entre el uso de redes sociales virtuales y el compromiso político de los jóvenes, de manera que estas redes podrían estar ayudando a reducir las diferencias en los patrones de comportamiento político tradicionales en función de variables tales como el nivel socioeconómico, la edad, la raza y la etnia.

En el ámbito español, el estudio de la participación política de los jóvenes también suscita gran interés; prueba de ello son las múltiples investigaciones realizadas a raíz del movimiento de los "indignados" (Monterde, 2012; Soler, 2013; Feixa, 2013; Nofre, 2013; Perondi, 2013). Estos y otros trabajos se enmarcan en un contexto caracterizado por tres circunstancias: la crisis económico-financiera de 2007 con la consiguiente desesperanza de una parte importante de la ciudadanía, especialmente entre los jóvenes; la deslegitimación de las instituciones políticas tradicionales; y, finalmente, el cambio tecnológico que ha supuesto Internet y las redes sociales. 
En este marco, la investigación llevada a cabo por el equipo de investigación IGOPnet de la Universidad Autónoma de Barcelona revela que los jóvenes se encuentran en un proceso de intensa politización. Este interés no se traduce en una mayor participación en la política convencional, pero sí en el desarrollo de nuevas formas de organización que aprovechan las posibilidades que brinda Internet (Berlinguer y Martínez, 2014). En el mismo sentido, el proyecto europeo MYPLACE, realizado en 16 países, llega a resultados similares para el ámbito de Cataluña: la mayoría de la gente joven está bastante o muy interesada por la política y se observa un fenómeno creciente de normalización de la participación política extra institucional (Feixa, 2014).

\section{Objetivos, hipótesis y metodología}

\section{Objetivos e hipótesis}

En este artículo hemos adoptado una definición de participación política fundamentalmente empírica, que engloba expresiones convencionales tales como la participación electoral y el posicionamiento ideológico, junto con nuevas formas de participación facilitadas y potenciadas por Internet y las redes sociales virtuales. En la práctica, dicha definición está necesariamente condicionada por las variables disponibles en el estudio del CIS que aquí se analiza. De esta forma, y tomando como población objeto de estudio a los jóvenes españoles de entre 15 y 29 años, el objetivo es analizar: 1) la relación existente entre el uso de Internet y la participación política y, en concreto, si un uso más frecuente de Internet se relaciona o no con una mayor participación política dentro y fuera de la red; 2) la posible relación entre la actividad política desarrollada en el espacio virtual y aquella otra relativa al espacio offline; y 3) las diferencias en términos de participación política según diversas variables sociodemográficas (sexo, edad, ocupación y nacionalidad). En definitiva, pretendemos contrastar las siguientes hipótesis:

- H1: Los jóvenes que utilizan Internet con mayor frecuencia son los que en mayor medida emplean la red con fines políticos.

- H2: Los jóvenes que desarrollan una intensa actividad política a través de Internet son también los más activos en la vida offline.

- H3: La participación de los jóvenes será diferente en función de distintas variables sociodemográficas como la nacionalidad, la edad, el sexo y la situación laboral. 


\section{Consideraciones metodológicas}

Se ha llevado a cabo un análisis secundario de los microdatos procedentes del estudio 2.889 del cIs, titulado "Actitudes hacia las tecnologías de la información y la comunicación". Se trata de una investigación elaborada por encargo del Instituto de la Juventud (INJUVE) en mayo de 2011, sobre una muestra de 1.419 jóvenes, de ambos sexos, residentes en España con edades comprendidas entre los 15 y los 29 años. $^{1}$ El objetivo de este estudio era recopilar información sobre las relaciones de los jóvenes con las nuevas tecnologías. Entre las variables usadas se incluyeron algunas que permiten analizar determinados aspectos de la utilización política de Internet. En concreto, en la Tabla $1^{2}$ se resumen las variables seleccionadas para la construcción de los indicadores del uso político, activo y pasivo, de la red. ${ }^{3}$

Una vez construidos estos indicadores (cuya elaboración se explica en el apartado de resultados), el análisis de los datos se ha centrado en la búsqueda de diferencias significativas en la utilización política de Internet según tres tipos de variables (véase Tabla 2): 1) aquellas relacionadas con la frecuencia y lugar de uso de Internet; 2) variables sociodemográficas; y 3) posicionamiento ideológico y comportamiento electoral, como indicadores de implicación política más allá del espacio virtual. Dado que la naturaleza nominal de las variables utilizadas impide la realización de un análisis factorial, se ha optado por aplicar un análisis exploratorio y descriptivo, basado en la búsqueda de relaciones a través de tablas de contingencia, atendiendo a los resultados de Chi-cuadrado y a los residuos tipificados corregidos, que permiten analizar no sólo la relación entre variables, sino también entre categorías concretas de las variables.

La aplicación de un análisis secundario a datos previamente existentes implica una serie de ventajas y desventajas. Entre las desventajas destaca la necesaria adaptación de los objetivos a los datos disponibles. Así, por ejem-

1 En el supuesto de muestreo aleatorio simple, con un nivel de confianza del 95,5\% (dos sigmas) y $\mathrm{P}=\mathrm{Q}$, el error real de muestreo es de $\pm 2,6 \%$. Fecha de realización del trabajo de campo: del 12 al 23 de mayo de 2011. Información ofrecida por el CIS: http://www.cis.es [21 de febrero de 2014].

2 Todas las tablas se encuentran en el Anexo del presente artículo (Nota del Editor).

3 Junto a las variables referidas al uso político de Internet, la Tabla 2 también incluye una última pregunta relativa a la recepción de mensajes a través del teléfono móvil. Se ha decido incluir esta variable en el análisis dada su íntima relación con el uso político de las nuevas tecnologías y la amplia expansión de la telefonía móvil entre la población joven. 
plo, en nuestro caso, ha sido necesario reducir el análisis de la participación política a un número limitado de las posibles formas que ésta puede adoptar.

Sin embargo, el análisis secundario también implica una serie de ventajas, al constituirse en una estrategia eficiente con la cual intentar responder a nuevas preguntas de investigación con base en datos obtenidos de acuerdo con otros objetivos y enfoques. Esta opción resulta especialmente relevante cuando se trabaja a partir de datos procedentes de instituciones públicas o centros de investigación (como es nuestro caso) que permiten acceder a información fiable obtenida a partir de muestras representativas de amplias poblaciones. Por ello, en el campo de estudio donde se sitúa nuestra investigación, otros autores han usado también el análisis secundario de datos previamente existentes (Schlozman et al., 2010; Morris y Moris, 2013; Anduiza et al., 2010).

\section{Resultados}

El uso de Internet está ampliamente generalizado en el colectivo objeto de estudio. Un $92 \%$ de los encuestados afirma haber utilizado Internet en los últimos tres meses (constituyen la submuestra que consideraremos usuarios de Internet y sobre la cual se realizarán los sucesivos análisis). Del total de usuarios, un $82 \%$ ocupa Internet diariamente (una o varias veces al día). Los porcentajes son también elevados para el uso de redes sociales. El hogar es el lugar habitual de conexión, y la mayoría (68\%) suele conectarse a Internet desde un ordenador particular (en la propia habitación u otro espacio privado). Los fines por los que se conectan son diversos si bien las actividades más frecuentes son: la búsqueda de información (82\%), la conexión a redes sociales $(80 \%)$ y la consulta del correo electrónico (76\%).

En cuanto a los usos políticos de Internet, tal y como señalamos anteriormente (véase Tabla 1), diferenciamos dos categorías: los usos que implican una participación activa y los usos pasivos, que básicamente consisten en la recepción de contenidos no solicitados. Respecto a los usos activos, no llega a la mitad (44\%) el porcentaje de jóvenes usuarios que busca información política en la red, y sólo el $24 \%$ lo hace una o más veces a la semana.

Algunos usos son especialmente minoritarios, como hacer una donación para una campaña, asociación u organización (7\%); contactar con algún/a político/a o algún partido político (4\%); y suscribirse a boletines o listas de distribución sobre temas de actualidad política (4\%). Otros usos presentan porcentajes algo más elevados, como contactar con alguna administración 
para protestar (11\%), firmar una petición o adherirse a alguna campaña o manifiesto $(14 \%)$ y consultar páginas web de partidos políticos o candidatos/as (16\%). Finalmente, destaca el porcentaje que afirma utilizar Internet para comunicarse con alguna asociación u organización (33\%) y para escribir comentarios en foros, blogs o webs sobre temas de actualidad, sociales o políticos (30\%).

Por lo que respecta a los usos pasivos, sólo una cuarta parte de los jóvenes usuarios ha entrado alguna vez en contacto con información política no buscada expresamente, lo que permite deducir que no es información política el principal contenido de la red. En proporciones similares se sitúa la recepción de correos electrónicos tanto con alguna crítica sobre un(a) candidato(a) o partido (22\%) como con un manifiesto o petición electrónica (22\%). Algo más elevada es la proporción de jóvenes que afirma haber recibido correos convocando a actos de protesta (33\%).

Finalmente, sólo el 11\% dice haber recibido correos electrónicos de apoyo a un(a) candidato(a) o partido, lo cual parece indicar una mayor presencia en la red de mensajes negativos que de apoyo a alguna opción política. Junto a estas variables, referidas directamente a Internet, se ha incluido en el análisis una última relativa a la telefonía móvil. En este sentido, sólo $17 \%$ de los encuestados afirma haber recibido SMS informando sobre manifestaciones, una proporción relativamente reducida si tenemos en cuenta el supuesto protagonismo de los móviles en la convocatoria de actos de protesta.

Con el objetivo de diferenciar el uso político intensivo de Internet del resto de posibilidades se ha optado por resumir la información disponible a través de la construcción de dos indicadores: relativos al uso activo y pasivo de la red. Para ello, se han sumado las respuestas afirmativas de cada actividad recogida en el cuestionario, de manera que los encuestados puedan ser clasificados según el número de actividades realizadas en tres categorías: los no participantes (ninguna actividad realizada), los moderadamente participantes (llevan a cabo de una a tres actividades) y los activistas políticos (los que efectúan de cuatro a seis). Los resultados pueden consultarse en la Tabla 3.

De esta forma, casi la mitad de los encuestados pueden clasificarse como no participantes: un $44 \%$ no tiene usos políticos activos y un $50 \%$ responde negativamente a las distintas opciones de uso político pasivo. En el extremo opuesto, el de los activistas políticos, se sitúan sólo el 13\% de los jóvenes si atendemos a los usos activos de Internet y un 11\% si atendemos a los pasivos.

Estos datos parecen confirmar el supuesto escaso interés de los jóvenes por la política que observan algunos autores (Zukin et al., 2006; Mindich, 
2005). Sin embargo, también pueden estar avalando la tesis mantenida por otros investigadores (Bennett, 2012; Coffé y Bolzendahl, 2010), según la cual la manera en la que los jóvenes conciben la política no tiene nada que ver con la concepción tradicional de la misma y, por consiguiente, tampoco tendrían validez los métodos que habitualmente se emplean para conocer su participación.

\section{Politica, frecuencia de uso de Internet y lugar de conexión}

La frecuencia e intensidad con que se utiliza Internet suele incluirse en las diferentes investigaciones como variable independiente a tener en cuenta (Valenzuela et al., 2012; Quintelier y Vissers, 2008). En nuestro caso, el primer objetivo es averiguar si los que ocupan con mayor frecuencia Internet también hacen un mayor uso político de la red. Igualmente, la encuesta analizada permite incluir otra variable relevante como es la referida al espacio en el que se ocupa Internet y, en concreto, a la posibilidad de utilizarlo en un espacio propio. Los resultados pueden consultarse en la Tabla $4 .{ }^{4}$

Ahí podemos observar que los usuarios más asiduos a Internet son también más activos políticamente en la red. Esta relación es estadísticamente significativa para los usos que hemos denominado pasivos (nunca hace un uso político pasivo de Internet el $42 \%$ de quienes lo ocupan diariamente, el $63 \%$ de los que lo usan semanalmente y el $76 \%$ de quienes usan la red con menor frecuencia).

En cierta forma, y como era previsible, la mera utilización frecuente de Internet parece incrementar la probabilidad de entrar en contacto con información política. Ahora bien, la relación es igualmente significativa en el caso de los usos activos (el porcentaje de los que nunca hacen usos políticos activos de Internet desciende hasta el 35\% para los usuarios más frecuentes, mientras que llega al 56\% en el caso de quienes realizan un uso semanal y al 67\% para los usuarios menos frecuentes).

Estos resultados son coherentes con los obtenidos al analizar el lugar de conexión a Internet. Así, los jóvenes que suelen utilizarla en un espacio no compartido presentan un uso político de la red (tanto pasivo como activo) significativamente mayor. De esta forma, aunque ni los datos obtenidos ni los

4 Tanto en la Tabla 4 como en las posteriores, la existencia de una relación significativa positiva entre las categorías de las variables (de acuerdo con los residuos tipificados corregidos) se ha resaltado con un sombreado oscuro, mientras que una relación significativa negativa se ha indicado con un sombreado claro. 
estadísticos construidos permiten afirmar que el uso de Internet incrementa la relación de los jóvenes con la política (la relación causal podría ser en sentido contrario), sí podemos hablar de una relación positiva entre el uso político de la red, así como de la frecuencia con que es utilizada y el hecho de contar con un espacio privado para la conexión.

Los datos del CIS también permiten comprobar la relación entre la frecuencia de uso de Internet y la implicación política fuera del espacio virtual. En concreto, para analizar la política no virtual contamos con dos variables: participación en las últimas elecciones generales y posicionamiento ideológico. Las diferencias en las respuestas a estas dos variables según la frecuencia y lugar de uso de Internet pueden consultarse en la Tabla 5.

En esta ocasión, la relación entre las diferentes variables no es tan unánime como en el caso de la política online. Sin embargo, podemos observar que continúa siendo significativamente mayor la expresión de una ideología entre los que ocupan con mayor frecuencia Internet (un $71 \%$ señala alguna ideología, frente al 59\% de quienes sólo utilizan la red semanalmente o el $58 \%$ de los que presentan una frecuencia menor), así como entre aquellos que se conectan en un espacio privado (el $72 \%$ afirma tener una ideología, frente al 63\% de los que utiliza Internet en ubicaciones compartidas).

En esta misma línea, son también los usuarios más asiduos de Internet y los que navegan desde un espacio propio quienes, en mayor medida, y de forma significativa de acuerdo con los residuos tipificados corregidos, afirman haber votado en las elecciones generales de 2008. En resumen, estos datos parecen indicar que aquellos jóvenes que usan de manera más intensiva Internet muestran también una mayor implicación política fuera del espacio virtual.

\section{Politica online y politica offline}

Como puede observarse en la Tabla 6, existe una relación estadísticamente significativa entre la utilización de Internet con fines políticos (pasivos y activos) y tanto expresar una ideología como votar. Con ello, podríamos afirmar que los jóvenes más implicados políticamente en el espacio virtual lo son también fuera de la red. Sólo un 34\% de los que expresan una ideología y un $35 \%$ de los que votaron en las elecciones generales de 2008 nunca hacen usos activos políticos de Internet (estos porcentajes se elevan hasta el 65\% para quienes no expresan una ideología y hasta el $56 \%$ para los que no quisieron votar en las últimas elecciones). La situación es semejante en el caso de los usos pasivos: si bien un $40 \%$ de los que expresan una ideología y un $42 \%$ de 
los que votaron niegan hacer estos usos; el porcentaje se eleva hasta el 68\% para los que no expresan ideología, y el 63\% para los que no quisieron votar.

Una vez más, no podemos deducir de estos datos que el uso político de la red sea la causa de una mayor politización de los jóvenes fuera del espacio virtual. Ahora bien, sí podemos hablar de una relación positiva entre ambas esferas de participación. De esta forma, y retomando los resultados anteriores, podemos concluir que son los jóvenes que hacen un uso más intensivo de la red quienes presentan un mayor uso político de la misma; los que más usan Internet son los que muestran una mayor implicación política offline; y existe una relación positiva entre un mayor uso político de la red y una mayor implicación política fuera de la misma.

\section{Uso político de Internet según variables sociodemográficas}

Respecto a las variables sociodemográficas, la teoría de la movilización plantea una progresiva disminución de las desigualdades en la medida en que Internet facilita la participación de los colectivos con mayor propensión a la exclusión política. Tal afirmación parece ser coherente, hasta cierto punto, con algunos de los resultados obtenidos (véase Tabla 7). Así, las diferencias tanto en materia de usos pasivos como activos entre jóvenes de nacionalidad española y de cualquier otra nacionalidad no resultan ser significativas.

Únicamente a través de los residuos tipificados corregidos podemos observar un menor uso político pasivo entre los jóvenes de nacionalidad no española, sin que haya diferencias significativas en materia de usos activos. Tampoco existe una relación estadísticamente significativa entre edad e intensidad de uso político de Internet (teniendo en cuenta que la edad contemplada en la encuesta va desde los 15 hasta los 29 años). Es también necesario acudir a los residuos tipificados corregidos para observar algunas relaciones entre categorías, como una mayor probabilidad entre los más jóvenes a la hora de no hacer ningún uso político activo de Internet.

Sin embargo, la relación entre usos políticos y la variable sexo sí presenta un valor de Chi-cuadrado significativo, aunque su correcta interpretación también requiere atender a los resultados de los residuos tipificados corregidos. De esta forma, si bien las diferencias entre hombres y mujeres no son significativas en cuanto a un uso político moderado o intensivo de Internet, sílo son en cuanto a que ese uso exista o no. Así, las mujeres en mayor medida que los hombres no desarrollan usos políticos, activos o pasivos, de la red. Finalmente, la situación laboral mantiene una relación claramente significativa 
con el número de usos políticos tanto pasivos como activos. Son los parados los que, en comparación con el resto de colectivos, desarrollan un menor uso político de la red (un 58\% nunca desarrolla usos activos y un $65 \%$ pasivos). En el extremo opuesto, son los jóvenes que estudian quienes hacen un mayor uso político de Internet; entre ellos se reduce hasta un 34\% el porcentaje de los que nunca realizan usos activos y hasta un $41 \%$ el de los que nunca se ven envueltos en usos pasivos.

\section{Conclusiones}

A lo largo de las páginas anteriores se ha analizado la participación de los jóvenes en política dentro y fuera del espacio virtual. Para el estudio de la política online, las distintas opciones de participación recogidas en la encuesta fueron agrupadas en dos categorías: formas activas y pasivas. Las primeras son aquellas que dependen de la voluntad expresa de los usuarios, las segundas llegan a los jóvenes de manera circunstancial. Unas y otras facilitan información sobre la implicación política de los jóvenes internautas y permiten contrastar las hipótesis de partida.

La primera hipótesis que nos planteamos es si, efectivamente, existe una relación positiva entre la frecuencia con que se utiliza Internet y el uso político de la red. Los datos confirman esta relación. Es decir, los jóvenes que con mayor frecuencia se conectan a Internet son los más activos políticamente en el ámbito online, tanto en aquellos usos que hemos denominado pasivos como en el caso de los activos. Estos resultados están en la línea de los hallazgos de autores como Calenda y Meijer (2009) o Valenzuela et al. (2012), quienes observan una correlación entre el tiempo de conexión y la participación política virtual. Hemos de anotar también, aunque no estuviera planteado en las hipótesis de partida, que los jóvenes que hacen un uso más intensivo de Internet no sólo muestran una mayor implicación política online, sino también fuera del espacio virtual. En resumen, podemos hablar de una relación positiva entre el uso de Internet y diferentes indicadores de participación política, aunque con nuestros datos resulte imposible determinar la dirección de una hipotética relación causal entre ambas dimensiones. De esta forma, los resultados podrían ser coherentes tanto con las tesis movilizadoras como con las normalizadoras. Para aclarar estas dinámicas serían necesarios estudios diacrónicos que permitan una mayor comprensión de los procesos de socialización política de los jóvenes.

En la segunda hipótesis tratamos de analizar la posible correspondencia entre la actividad política online y offine. En este caso, aunque los resulta- 
dos son menos concluyentes que los referidos a la primera hipótesis, también permiten hablar de una relación positiva entre ambos espacios; es decir, los jóvenes que son más activos políticamente en el ámbito virtual lo son también en la vida offline. Una vez más, con los datos analizados no podemos afirmar que Internet esté fomentando la participación política de los jóvenes fuera de la red, ya que sólo permiten hablar de una correlación pero no de una relación causal. Estos resultados están en consonancia con los obtenidos por otros autores como Calenda y Meijer (2009), Oser et al. (2013) o Robles et al. (2012).

La tercera hipótesis planteaba una participación política en Internet diferencial con base en distintas variables sociodemográficas, en concreto: nacionalidad, situación laboral, edad y sexo. Respecto a la nacionalidad, los resultados no reflejan diferencias significativas; es decir, los jóvenes de nacionalidad española y los de otras nacionalidades presentan una implicación política similar. Sin embargo, las diferencias son claramente significativas en función de la variable situación laboral.

En este sentido, los que muestran una mayor implicación política en Internet son los estudiantes, mientras que los jóvenes en situación de desempleo son los que hacen un menor uso político de la red. Esta diferencia resulta especialmente relevante, dados los altos niveles actuales de desempleo juvenil en España y sus posibles efectos en términos de exclusión social (Lasheras y Pérez, 2014). En cuanto a la edad, nuestros resultados parecen coincidir con gran parte de las investigaciones previas, que suelen destacar un aumento de la implicación política según se incrementa la edad (Vilas, 2003). Aún así, el estadístico Chi-cuadrado no es significativo y únicamente el análisis de los residuos tipificados corregidos nos permite hablar de una menor implicación política de los más jóvenes.

Finalmente, respecto a la variable sexo, los resultados apuntan hacia un efecto limitado, pero aún significativo, de esta variable, en la medida en que las mujeres parecen desarrollar un menor uso político de Internet que los hombres. Si analizamos de forma desagregada cada uno de los usos resumidos en los indicadores, se observa que mientras los chicos son más activos que las chicas en algunas de las actividades, por ejemplo, obtener información política o consultas de páginas web de partidos políticos, las mujeres lo son en otras, tales como suscribirse a una lista de distribución o contactar con alguna administración para protestar. Estas diferencias según actividades han sido también observadas por otros investigadores, quienes destacan un mayor alejamiento de las mujeres de la política formal e institucional (Roker, 2008). 
En nuestro trabajo partimos, en gran medida, de una concepción clásica de la participación política y, desde esta orientación, los resultados parecen confirmar el déficit de participación de los jóvenes, tanto online como offline. Sin embargo, aunque es relativamente reducido el porcentaje de jóvenes que tienen una participación política intensiva en Internet, estos sí parecen hacer un amplio aprovechamiento de las opciones que les ofrece la red para su actividad política, que se relaciona igualmente con una implicación política en el espacio no virtual.

Dada la naturaleza de nuestros datos, no podemos afirmar que el uso de Internet esté fomentando, en algunos casos, una mayor implicación política, ya que el sentido de la relación causal podría ser la opuesta (los más interesados en política pueden sentir una mayor motivación para usar Internet y las herramientas de comunicación e información que pone a su disposición). De hecho, la relación estadísticamente significativa entre la actividad política virtual y offline parece situar nuestros resultados más próximos a la teoría de la normalización, según la cual Internet es una forma alternativa de participación especialmente útil para aquellos que ya desarrollan una actividad política previa. En todo caso, sería necesario ampliar la investigación con datos de naturaleza diacrónica o cualitativa, que permitan una mayor comprensión de estos procesos.

Finalmente, nuestros resultados parecen incongruentes con una supuesta politización de los jóvenes españoles que otros estudios destacan (Berlinguer y Martínez, 2014; Feixa, 2014). Sin embargo, parte de las discrepancias pueden situarse en una diferente concepción de la participación política (más reducida en nuestro caso y más amplia en otros estudios). Igualmente, sería necesario replicar investigaciones como la que aquí se presenta con nuevos datos que recojan los posibles efectos de las movilizaciones políticas desarrolladas en España durante los últimos años. 


\section{Anexo}

\section{Tabla 1}

\section{Variables sobre uso político de Internet recogidas en el cuestionario}

\begin{tabular}{|c|c|}
\hline \multirow{4}{*}{ Uso político activo } & Frecuencia de uso de Internet para obtener información política. \\
\hline & Consulta de páginas web de un partido político o candidato/a. \\
\hline & $\begin{array}{l}\text { Suscripción a boletines o listas de distribución sobre actualidad } \\
\text { política. }\end{array}$ \\
\hline & $\begin{array}{l}\text { Actividades políticas a través de Internet: contactar con algún } \\
\text { político/a o partido político; contactar con alguna administración } \\
\text { para quejarse; comunicarse con alguna asociación u organización; } \\
\text { realizar una donación para una campaña, asociación u organización; } \\
\text { escribir comentarios en algún foro, blog o página web; firmar una } \\
\text { petición o adherirse a alguna campaña o manifiesto. }\end{array}$ \\
\hline \multirow{3}{*}{ Uso político pasivo } & $\begin{array}{l}\text { Recepción de correos electrónicos: de apoyo a un candidato/a } \\
\text { o partido; con alguna crítica sobre un político/a o partido; } \\
\text { convocando a una manifestación o acto de protesta; con un } \\
\text { manifiesto o petición. }\end{array}$ \\
\hline & $\begin{array}{l}\text { Recepción o acceso involuntario a información sobre política o } \\
\text { campañas electorales. }\end{array}$ \\
\hline & Recepción de SMS informando sobre alguna manifestación. \\
\hline
\end{tabular}

Fuente: Elaboración propia a partir del cuestionario del estudio núm. 2.889 del CIS. 


\section{Tabla 2}

\section{Variables explicativas recogidas en el cuestionario}

\begin{tabular}{|c|c|}
\hline \multirow{4}{*}{$\begin{array}{l}\text { 1. Frecuencia y } \\
\text { lugar de uso de } \\
\text { Internet }\end{array}$} & Uso de Internet en los últimos tres meses. \\
\hline & Frecuencia de uso de Internet. \\
\hline & Uso habitual de Internet en un espacio propio. \\
\hline & Frecuencia de uso de las redes sociales en Internet. \\
\hline \multirow{4}{*}{$\begin{array}{l}\text { 2. Variables } \\
\text { sociodemográficas }\end{array}$} & Edad \\
\hline & Sexo \\
\hline & Situación laboral \\
\hline & Nacionalidad \\
\hline \multirow{2}{*}{$\begin{array}{l}\text { 3. Posicionamiento } \\
\text { ideológico y } \\
\text { comportamiento } \\
\text { electoral }\end{array}$} & Posicionamiento ideológico \\
\hline & Comportamiento electoral en las últimas elecciones generales \\
\hline
\end{tabular}

Fuente: Elaboración propia a partir del cuestionario del estudio núm. 2889 del CIS.

\section{Tabla 3}

Grupos de intensidad de uso político de la red (Base: usuarios de Internet en los tres últimos meses)

\begin{tabular}{lcc}
\hline & Grupos de intensidad de uso & Porcentaje \\
\hline \multirow{3}{*}{ Usos activos } & No participantes (ningún uso) & 44,0 \\
\cline { 2 - 3 } & Moderados (de 1 a 3) & 43,6 \\
\cline { 2 - 3 } & Activistas (de 4 a 6) & 12,5 \\
\cline { 2 - 3 } & Total & 100,0 \\
\cline { 2 - 3 } Usos pasivos & No participantes (ningún uso) & 49,9 \\
\cline { 2 - 3 } & Moderados (de 1 a 3) & 39,3 \\
\cline { 2 - 3 } & Activistas (de 4 a 6) & 10,9 \\
\hline
\end{tabular}

Fuente: Elaboración propia a partir del estudio núm. 2.889 del CIS. 


\section{Tabla 4}

Intensidad de uso político de la red según frecuencia y lugar de conexión (\%) (Base: usuarios de Internet en los tres últimos meses)

\begin{tabular}{|c|c|c|c|c|c|c|}
\hline & & \multicolumn{3}{|c|}{ Frecuencia de uso de Internet } & \multicolumn{2}{|c|}{$\begin{array}{c}\text { Conexión habitual en } \\
\text { un espacio propio }\end{array}$} \\
\hline & & Diaria & Semanal & Menor & Sí & No \\
\hline \multirow{7}{*}{$\begin{array}{l}\text { Usos } \\
\text { activos }\end{array}$} & Ninguno & 34,8 & 55,7 & 66,7 & 34,3 & 48,5 \\
\hline & De 1 a 3 & 49,6 & 38,5 & 33,3 & 49,7 & 42,9 \\
\hline & De 4 a 6 & 15,6 & 5,7 & 0,0 & 16,0 & 8,5 \\
\hline & Total & 100,0 & 100,0 & 100,0 & 100,0 & 100,0 \\
\hline & \multicolumn{4}{|c|}{ Valor Chi-cuadrado 50,410; significación 0,000 } & \multicolumn{2}{|c|}{$\begin{array}{l}\text { Valor Chi-cuadrado } \\
28,652 \text {; significación } \\
0,000\end{array}$} \\
\hline & & \multicolumn{3}{|c|}{ Frecuencia de uso de Internet } & \multicolumn{2}{|c|}{$\begin{array}{c}\text { Conexión habitual en } \\
\text { un espacio propio }\end{array}$} \\
\hline & & Diaria & Semanal & Menor & Sí & No \\
\hline \multirow{5}{*}{$\begin{array}{l}\text { Usos } \\
\text { pasivos }\end{array}$} & Ninguno & 42,1 & 63,0 & 76,2 & 41,4 & 57,1 \\
\hline & De 1 a 3 & 44,6 & 30,7 & 23,8 & 45,6 & 33,7 \\
\hline & De 4 a 6 & 13,3 & 6,3 & 0,0 & 13,0 & 9,3 \\
\hline & Total & 100,0 & 100,0 & 100,0 & 100,0 & 100,0 \\
\hline & \multicolumn{4}{|c|}{ Valor Chi-cuadrado 46,536; significación 0,000} & \multicolumn{2}{|c|}{$\begin{array}{l}\text { Valor Chi-cuadrado } \\
27,578 \text {; significación } \\
0,000\end{array}$} \\
\hline
\end{tabular}

Fuente: Elaboración propia a partir del estudio núm. 2889 del CIS. 


\section{Tabla 5}

\section{Ideología y participación en las elecciones según frecuencia y lugar de conexión a Internet $(\%)$}

\begin{tabular}{|c|c|c|c|c|c|c|}
\hline & & \multicolumn{3}{|c|}{ Frecuencia uso de Internet } & \multicolumn{2}{|c|}{$\begin{array}{l}\text { Conexión en espacio } \\
\text { propio }\end{array}$} \\
\hline & & Diaria & Semanal & Menor & Sí & No \\
\hline \multirow{6}{*}{$\begin{array}{l}\text { Expresa una } \\
\text { ideología } \\
\text { Base: usuarios de } \\
\text { Internet en los tres } \\
\text { últimos meses }\end{array}$} & Sí & 71,4 & 59,3 & 57,9 & 72,0 & 63,0 \\
\hline & No & 28,6 & 40,7 & 42,1 & 28,0 & 37,0 \\
\hline & Total & 100,0 & 100,0 & 100.0 & 100,0 & 100,0 \\
\hline & \multicolumn{4}{|c|}{$\begin{array}{l}\text { Valor Chi-cuadrado 12,884; significación } \\
0,002\end{array}$} & \multicolumn{2}{|c|}{$\begin{array}{l}\text { Valor Chi-cuadrado } \\
10,242 \text {; significación } \\
0,001\end{array}$} \\
\hline & & \multicolumn{3}{|c|}{ Frecuencia uso de Internet } & \multicolumn{2}{|c|}{$\begin{array}{l}\text { Conexión en espacic } \\
\text { propio }\end{array}$} \\
\hline & & Diaria & Semanal & Menor & Sí & No \\
\hline \multirow{5}{*}{$\begin{array}{l}\text { Voto en las últimas } \\
\text { elecciones generales } \\
\text { Base: usuarios de } \\
\text { Internet en los } \\
\text { últimos tres meses } \\
\text { que tenían edad } \\
\text { para votar }\end{array}$} & Votó & 64,9 & 50,4 & 61,3 & 65,0 & 57,9 \\
\hline & No pudo & 14,1 & 25,2 & 19,4 & 14,6 & 18,8 \\
\hline & No quiso & 21,0 & 24,4 & 19,4 & 20,4 & 23,3 \\
\hline & Total & 100,0 & 100,0 & 100,0 & 100,0 & 100,0 \\
\hline & \multicolumn{4}{|c|}{$\begin{array}{l}\text { Valor Chi-cuadrado 12,394; significación } \\
0,015\end{array}$} & \multicolumn{2}{|c|}{$\begin{array}{l}\text { Valor Chi-cuadrado } \\
4,194 ; \text { significación } \\
0,123\end{array}$} \\
\hline
\end{tabular}

Fuente: Elaboración propia a partir del estudio núm. 2889 del CIS. 


\section{Tabla 6}

Uso político de la red según ideología y participación electoral (\%)

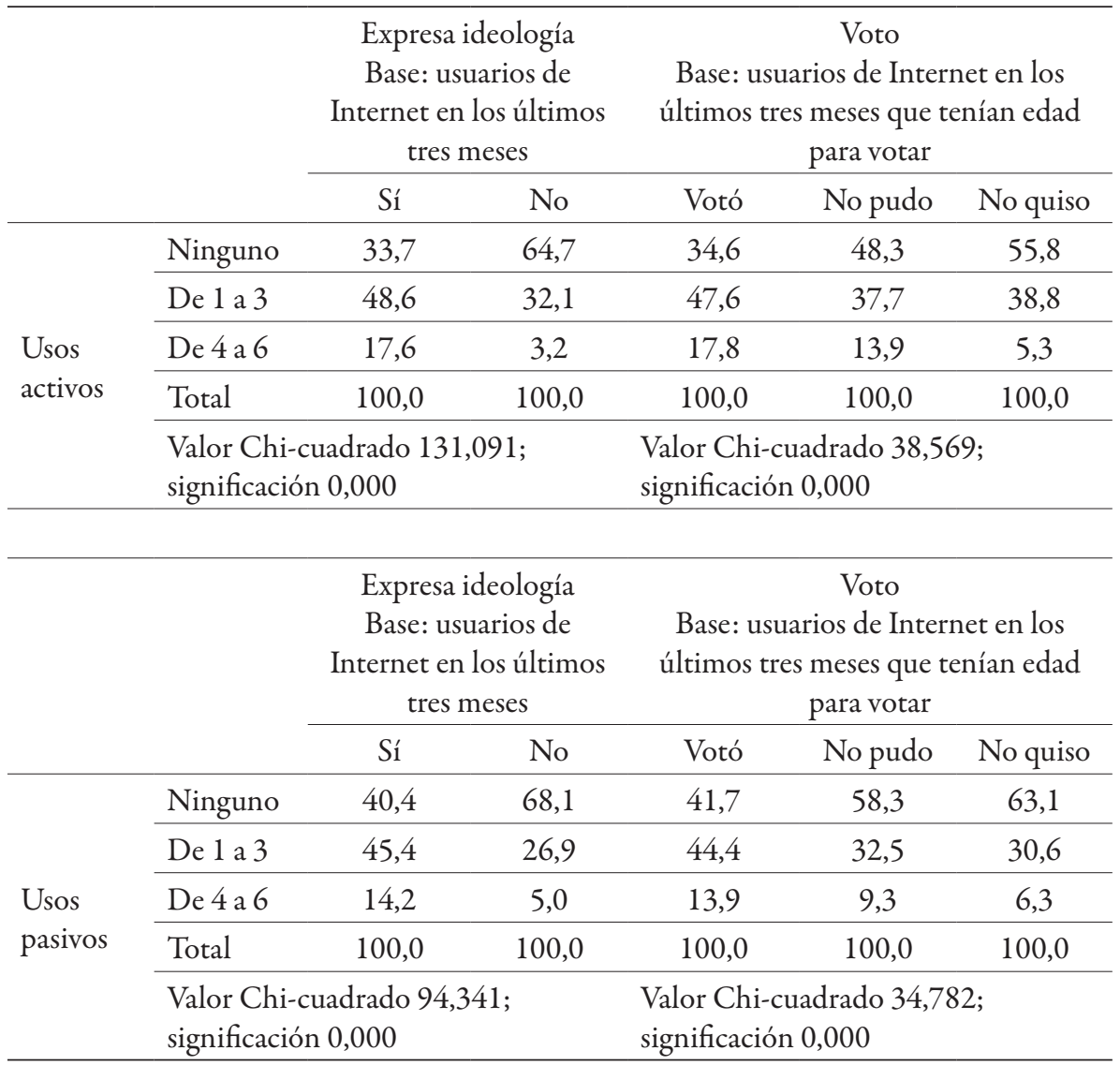

Fuente: Elaboración propia a partir del estudio núm. 2.889 del CIS. 


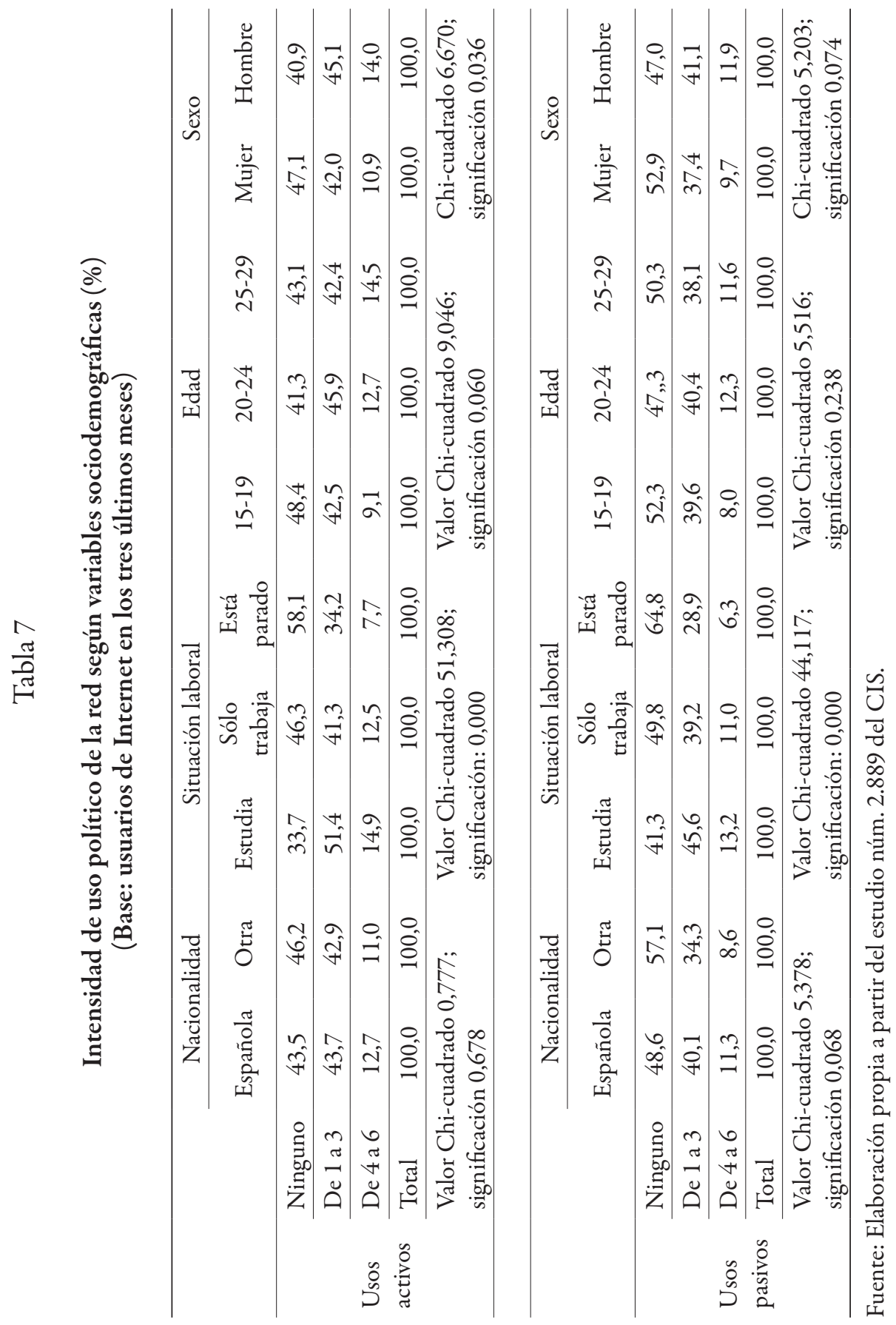


Convergencia Revista de Ciencias Sociales, núm. 69, 2015, Universidad Autónoma del Estado de México

\section{Bibliografía}

Anduiza, Eva, Gallego, Aina y Marta Cantijoch (2010), "Online Political Participation in Spain: The Impact of Traditional and Internet Resources”, en Journal of Information Technology \& Politics, vol. 7, núm. 4, Londres: Routledge.

Bakker, Tom P. y Claes H. de Vreese (2011), "Good News for the Future? Young People, Internet Use, and Political Participation”, en Communication Resarch, vol. 38, núm. 4, Thousand Oaks, California: Sage.

Banaji, Shakuntala y David Buckingham (2010), "Young People, the Internet, and Civic Participation: An Overview of Key Findings from the CivicWeb Project", en International Journal of Learning and Media, vol. 2, núm. 1, Cambridge, Massachusetts: MIT Press.

Bauman, Zygmunt (2007), Tiempos líquidos. Vivir en una época de incertidumbre, Barcelona: Tusquets.

Baumgartner, Jody C. y Jonathan S. Morris (2010), "MyFaceTube Politics: Social Networking Web Sites and Political Engagement of Young Adults", en Social Science Computer Review, vol. 28, núm. 1, Thousand Oaks, California: Sage.

Bennett, W. Lance (2012), “The personalization of politics: Political identity, social media, and changing patternsof participation", en The ANNALS of the American Academy of Political and Social Science, vol. 644, núm. 1, Thousand Oaks, California: Sage.

Bennett, W. Lance, Wells, Chris y Deen Freelon (2011), "Communicating civic engagement: Contrasting models of citizenship in the youth web sphere”, en Journal of Communication, vol. 61, núm. 5, Hoboken, New Jersey: Wiley-Blackwell.

Berlinguer, Marco y Rubén Marínez (2014), "Desconfiados: suspendidos entre búsqueda, resignación y revuelta. Una situación inestable”, en Equipo IDOPnet, Jóvenes, Internet y politica, Madrid: Centro Reina Sofía sobre Adolescencia y Juventud y Fundación de Ayuda contra la Drogadicción (FAD).

Bode, Leticia (2012), "Facebooking it to the polls: A study in online social networking and political behavior”, en Journal of Information Technology \& Politics, vol. 9, núm. 4, Londres: Routledge.

Calenda, Davide y Albert Meijer (2009), "Young people, the Internet and political participation. Findings of a web survey in Italy, Spain and The Netherlands", en Information, Communication \& Society, vol. 12, núm. 6, Londres: Routledge.

Castells, Manuel (2012), Redes de indignación y esperanza, Madrid: Alianza.

Coffé, Hilde y Catherine Bolzendahl (2010), "Same Game, Different Rules? Gender Differences in Political Participation”, en Sex Roles, vol. 62, núms. 5-6, Nueva York: Springer.

Dahlgren, Peter (2011), "Young citizens and political participation. Online media and civic cultures”, en Taiwan Journal of Democracy, vol. 7, núm. 2, Taipei: Taiwan Foundation for Democracy.

Dimitrova, Daniela V. y Dianne Bystrom (2013), “The effects of social media on political participation and candidate image evaluations in the 2012 Iowa Caucuses", en American Behavioral Scientist, vol. 57, núm. 11, Thousand Oaks, California: Sage. 
Ekström, Mats, Olsson, Tobias y Adam Shehata (2014), "Spaces for public orientation? Longitudinal effects of Internet use in adolescence", en Information, Communication \& Society, vol. 17, núm. 2, Londres: Routledge.

Feixa, Carles (2013), “Crónicas del 15M del campamento al agora”, en Feixa, C. y Nofre Jordi, Generación indignada. Topias y Utopias del 15M, Madrid: Milenio.

Feixa, Cales (2014), “Juventud y participación política en la era digital: estado del arte versus artes del estado", en Equipo IDOPnet, Jóvenes, Internet y politica, Madrid: Centro Reina Sofía sobre Adolescencia y Juventud y FAD.

Furlong, Andy y Fred Cartmel (2007), Youngpeople and social change: individualisation and risk in late modernity, Maidenhead: Open University Press.

Gil de Zúñiga, Homero, Jung, Nakwon y Sebastián Valenzuela (2012), “Social media use for news and individuals' social capital, civic engagement and political participation”, en Journal of Computer-Mediated Communication, vol. 17, núm. 3, Hoboken, New Jersey: Wiley-Blackwell.

Gil de Zúñiga, Homero et al. (2010), "Digital democracy: Reimagining pathways to political participation”, en Journal of Information Technology \& Politics, vol. 7, núm. 1, Londres: Routledge.

Hirzalla, Fadi; van Zoonen, Liesbet y Jan de Ridder (2011), "Internet Use and Political Participation: Reflections on the Mobilization/Normalization Controversy", en The Information Society, vol. 27, núm.1, Londres: Routledge.

Lasheras, Rubén y Begoña Pérez (2014), “Jóvenes, vulnerabilidades y exclusión social: impacto de la crisis y debilidades del sistema de protección social”, en Zerbitzuan. Revista de Servicios Sociales, núm. 57, Vitoria: Servicio Central de Publicaciones del Gobierno Vasco.

Lee, Nam-Jin, Shah, Dhavan V. y Jack M. McLeod (2013), "Processes of political socialization: A communication mediation approach to youth civic engagement", en Communication Research, vol. 40, núm. 5, Thousand Oaks, California: Sage.

Livingstone, Sonia (2007), “The challenge of engaging youth online: contrasting producers' and teenagers' interpretations of websites”, en European Journal of Communication, vol. 22, núm. 2, Thousand Oaks, California: Sage.

Livingstone, Sonia (2010), "Interactivity and participation on the Internet. A critical appraisal of the online invitation to young people", en Peter Dahlgren [ed.], Young citizens and new media: learning for democratic participation, Londres: Routledge.

Loader, Brian D., Vromen, Ariadne y Michael A. Xenos (2014), “The networked young citizen: social media, political participation and civic engagement", en Information, Comunication \& Society, vol. 17, núm. 2, Londres: Routledge.

Macafee, Timothy y J.J. De Simone (2012), "Killing the Bill Online? Pathways to Young People's Protest Engagement via Social Media”, en Cyberpsychology, Behavior, and Social Networking, vol. 15, núm. 11, New Rochelle, Nueva York: Mary Ann Liebert, Inc.

Mindich, David T. Z. (2005), Tuned Out. Why Americans Under 40 Don't Follow the News, Oxford: Oxford University Press.

Monterde, Arnau (2012), Tecnopolitica, Internet y R-evoluciones. Sobre la centralidad de las redes digitales en el \#15M, Barcelona: Icaria. 
Morozov, Evgeny (2009), "Iran: Downside to the 'Twitter revolution”, en Dissent, vol. 56, núm. 4, Nueva York: University of Pennsylvania Press.

Morris, David S. y Jonathan S. Morris (2013), “Digital Inequality and Participation in the Political Process. Real or Imagined?”, en Social Science Computer Review, vol. 31, núm. 5, Thousand Oaks, California: Sage.

Nam, Taewoo (2012), "Dual effects of the Internet on political activism: Reinforcing and mobilizing”, en Government Information Quarterly, vol. 29, núm. 1, Amsterdam: Elsevier.

Nofre, J. (2013), "Del pacto social a la indignación: Geografía(s) de la \#Spanish Revolution”, en Feixa, C. y Jordi Nofre, Generación indignada. Topias y Utopias del 15M, Madrid: Milenio.

Oser, Jennifer; Hooghe, Marc y Sofie Marien (2013), “Is Online Participation Distinct from Offline participation? A Latent Class Analysis of Participation Types and Their Stratification", en Political Research Quarterly, vol. 66, núm. 1, Thousand Oaks, California: Sage.

Perondi, Mauricio (2013) “¿Nació el 15M en Porto Alegre?”, en Feixa, C. y Jordi Nofre, Generación indignada. Topias y Utopias del 15M, Madrid: Milenio.

Quintelier, Ellen y Sara Vissers (2008), “The Effect of Internet Use on Political Participation. An Analysis of Survey Results for 16-Year-Olds in Belgium”, en Social Science Computer Review, vol. 26, núm. 4, Thousand Oaks, California: Sage.

Robles, José M., De Marco, Stefano y Mirko Antino (2012), “Activating Activists. The links between political participation and digital political participation", en Information, Communication and Society, vol. 16, núm. 6, Londres: Routledge.

Roker, Debi (2008), "Young women and social action in the UK”, en Anita Harris [ed.] Next wave cultures: feminism, subcultures, activism, Nueva York: Routledge.

Scheufele, Dietram A. y Matthew C. Nisbet (2002), "Being a citizen online: New opportunities and dead ends", en Press/Politics, vol. 7, núm. 3, Thousand Oaks, California: Sage.

Schlozman, Kay L.; Verba, Sidney y Henry E. Brady (2010), "Weapon of the Strong? Participatory Inequality and the Internet”, en Perspectives on Politics, vol. 8, núm. 2, Cambridge: Cambridge University Press.

Sloam, James (2012), "New Voice, Less Equal: The Civic and Political Engagement of Young People in the United States and Europe”, en Comparative Political Studies, vol. 47, núm. 5, Thousand Oaks, California: Sage.

Sloam, James (2014), “'The outraged young': young Europeans, civic engagement and the new media in a time of crisis", en Information, Communication \& Society, vol. 17, núm. 2, Londres: Routledge.

Soler, Roger (2013), Democràcia, participació i joventut. Una anàlisi de l'enquesta de participació politica 201, Barcelona: Generalitat de Catalunya.

Valenzuela, Sebastián; Arriagada, Arturo y Andrés Scherman (2012), “The Social Media Basis of Youth Protest Behavior: The Case of Chile”, en Journal of Communication, vol. 62, núm. 2, Hoboken, New Jersey: Wiley-Blackwell. 
Valenzuela, Sebastián; Park, Namsu y Kerk F. Kee (2009), "Is there social capital in a social network site?: Facebook use and college students' life satisfaction, trust, and participation", en Journal of Computer-Mediated Communication, vol. 14, núm. 4, Hoboken, New Jersey: Wiley-Blackwell.

Vissers, Sara y Dietlind Stolle (2014), “The Internet and new modes of political participation: online versus offline participation”, en Information, Communication \& Society, vol. 17, núm. 8, Londres: Routledge.

Vitak, Jessica et al. (2011), “It's complicated: Facebook users' political participation in the 2008 election”, en Cyberpsychology, Behavior and Social Networking, vol. 14, núm. 3, New Rochelle, Nueva York: Mary Ann Liebert, Inc.

Xenos, Michael; Vromen, Ariadne y Brian D. Loader (2014), “The great equalizer? Patterns of social media use and youth political engagement in three advanced democracies", en Information, Communication \& Society, vol. 17, núm. 2, Londres: Routledge.

Yamamoto, Masahiro y Matthew J. Kushin (2014), "More harm than good? Online media use and political disaffection among college students in the 2008 election", en Journal of Computer-Mediated Communication, vol. 19, núm. 3, Hoboken, New Jersey: WileyBlackwell.

Zhang, Weiwu; Seltzer, Trent y Shannon L. Bichard (2013), "Two sides of the coin: Assessing the influence of social network site use during the 2012 U.S. Presidential campaign", en Social Science Computer Review, vol. 31, núm. 5, Thousand Oaks, California: Sage.

Zhou, Yushu y Bruce Pinkleton (2012), "Modeling the Effects of Political Information Source Use and Online Expression on Young Adults' Political Efficacy”, en Mass Communication and Society, vol. 15, núm. 5, Londres: Routledge.

Zukin, Cliff et al. (2006), A new engagement? Political participation, civic life, and the changing American citizen, Nueva York: Oxford University Press.

\section{Recursos electrónicos}

Borge, Rosa, Cardenal, Ana S. y Claudia Malpica (2012), "El impacto de Internet en la participación política: revisando el papel del interés político", en Arbor, vol. 188, núm. 756. Disponible en: http://arbor.revistas.csic.es/index.php/arbor/article/ view/1497 [10 de febrero de 2015].

De Marco, Stefano y José M. Robles (2012), “Uso de los blogs políticos: análisis de algunos factores determinantes”, en Arbor, vol. 188, núm. 756. Disponible en: http://arbor. revistas.csic.es/index.php/arbor/article/viewFile/1494/1505 [10 de febrero de 2015].

Vilas, José (2003), “Bienestar individual e interés por la política”, en RIPS, vol. 2, núm. 2. Disponible en: http://dspace.usc.es/handle/10347/8204 [10 de febrero de 2015]. 
Eva Espinar Ruiz. Doctora en Sociología y profesora en el Departamento de Sociología II de la Universidad de Alicante, España. Investigadora y Secretaria Académica del Instituto Interuniversitario de Desarrollo Social y Paz (IUDESP) de esta misma universidad. Gran parte de su actividad investigadora se encuadra en torno a los estudios de género y la sociología de la comunicación. Publicaciones recientes: "Riesgos medioambientales en los espacios informativos: análisis cualitativo de la televisión española”, en Estudios sobre el mensaje periodistico, 18(2) (2012); "El periodismo de paz como paradigma de comunicación para el cambio social: características, dimensiones y obstáculos", en $C I C$, núm. 17 (2012); "Gender portrayals in food commercials: A content analysis of Spanish television advertisements", en Observatorio $\left(O B S^{*}\right), 6(4)$ (2012).

María José González Río. Doctora en Sociología y Profesora Titular en el Departamento de Sociología II de la Universidad de Alicante, España. Entre sus principales líneas de investigación destacan los estudios sobre juventud. En esta materia ha dirigido el proyecto Juventud y nuevas tecnologías. El impacto de Internet en la vida cotidian a de los jóvenes financiado mediante ayuda de $\mathrm{I}+\mathrm{D}+\mathrm{I}$. Ha participado en otros proyectos de carácter internacional, nacional y autonómico sobre ésta y otras materias: género, técnicas biográficas y comunicación, dando lugar a diversas aportaciones, insertas en capítulos de libros y artículos de revistas. Recientemente ha publicado con otras autoras: "Las carreras académicas de mujeres y hombres en la Universidad de Alicante", en Sociología del Trabajo, núm. 80 (2014). Con Eva Espinar ha publicado: "Jóvenes en las redes sociales virtuales: un análisis exploratorio de las diferencias de género", en Feminismo/s, núm. 14 (2009); y "Jóvenes conectados: las experiencias de los jóvenes con las nuevas tecnologías”, en RES, núm. 9 (2008).

Recepción: 28 de mayo de 2014.

Aprobación: 17 de febrero de 2015. 